\title{
Next-generation sequencing in the diagnosis and minimal residual disease assessment of acute myeloid leukemia
}

Haematologica 2019

Volume 104(5):868-871

\section{Correspondence:}

ROSS L. LEVINE

leviner@mskcc.org

PETER J.M. VALK

p.valk@erasmusmc.nl

Received: February 1, 2019.

Accepted: February 14, 2019.

Pre-published: March 28, 2019.

doi:10.3324/haematol.2018.205955

Check the online version for the most updated information on this article, online supplements, and information on authorship \& disclosures: www.haematologica.org/content/104/5/868

(C)2019 Ferrata Storti Foundation

Material published in Haematologica is covered by copyright. All rights are reserved to the Ferrata Storti Foundation. Use of published material is allowed under the following terms and conditions:

https://creativecommons.org/licenses/by-nc/4.0/legalcode. Copies of published material are allowed for personal or internal use. Sharing published material for non-commercial purposes is subject to the following conditions:

https://creativecommons.org/licenses/by-nc/4.0/legalcode, sect. 3. Reproducing and sharing published material for commercial purposes is not allowed without permission in writing from the publisher.

\section{Ross L. Levine and Peter J.M. Valk}

${ }^{1}$ Human Oncology and Pathogenesis Program, Memorial Sloan Kettering Cancer Center, New York, NY, USA and '2Department of Hematology, Erasmus University Medical Center, Rotterdam, the Netherlands

\section{Introduction}

Risk-stratification of acute myeloid leukemia (AML) based on recurrent somatic abnormalities has evolved substantially in recent years, as illustrated by the current 2017 European LeukemiaNet (ELN) risk stratification. ${ }^{1}$ These 2017 ELN AML risk stratification recommendations are based on (cyto)genetic aberrations, including hotspot mutations such as those in NPM1, but also small insertions, deletions and point mutations that occur throughout TP53, RUNX1 and ASXL1, the latter being associated with adverse outcome. ${ }^{1}$ Next-generation sequencing (NGS) enables reliable detection of patient-specific mutations covering complete genes in molecularly heterogeneous diseases such as AML. NGS should, therefore, be incorporated in the routine work-up of preferably bone marrow specimens for accurate risk stratification in AML. Since risk assessment according to 2017 ELN recommendations only requires knowledge of the status of a handful of wellknown driver mutations, ${ }^{1}$ targeted NGS, easily reaching a sensitivity of $1-2 \%$, is currently the most appropriate and cost-effective approach for routine testing in AML. Targeted NGS using a variety of gene panels has been successfully introduced in routine clinical laboratories; however, several challenges remain.

\section{Gene panels}

A number of commercially available gene panels focusing on genes frequently mutated in myeloid malignancies have been introduced, e.g., the Illumina TruSight Myeloid panel, the Archer VariantPlex Core Myeloid panel, the Human Myeloid Neoplasms OIASeq DNA Panel and the AmpliSeq for Illumina Myeloid panel among many others. As expected, these panels contain all genes relevant for the 2017 ELN classification and show an enormous overlap in additional mutational hotspots and complete coverage of genes frequently mutated in myeloid diseases. In addition to these commercial panels, gene panels can be easily configured to meet local requirements. For instance, if AML patients are classified locally according to 2017 ELN, only NPM1, CEBPA, FLT3, RUNX1, ASXL1, and TP53 need to be included in a small and cost-effective gene panel. These types of NGS-based assays are now emerging. ${ }^{2}$ Importantly, both commercial NGS-based assays and those developed in-house as well as downstream analyses should be thoroughly validated locally before implementation in daily practice can be considered.

Some genes are particularly difficult to sequence with NGS using gene panels. Bi-allelic mutations in CEBPA characteristically confer a favorable outcome in patients with AML. ${ }^{1}$ CEBPA is a GC-rich gene which is notoriously difficult to amplify by polymerase chain reaction (PCR) and sequence, and should be given special attention when incorporated in a gene panel. Although some commercial NGS gene panel protocols do now successfully include this single exon gene, other NGS approaches, such as capture-based NGS or custom panels for CEBPA mutation detection could be considered. FLT3 internal tandem duplications (ITD) can be reliably determined by fragment-length PCR following standardized protocols ${ }^{3}$ however, the size of the FLT3 ITD and the duplication itself make it challenging to sequence the variably-sized amplicons appropriately by NGS and subsequently to analyze the FLT3 ITD by sequence alignment to reference sequences. Moreover, the 2017 ELN recommendations require assessment of the size of the FLT3 ITD clone. ${ }^{1}$ NPM1-mutant AML cases with high FLT3 ITD/FLT3 wildtype ratios $(>0.5)$ are considered at intermediate risk, whereas NPM1 wildtype AML cases with high FLT3 ITD/FLT3 wildtype ratios are seen as adverse. Standardized NGS-based protocols need to be developed not only for the detection of FLT3 ITD, but also for the quantification of FLT3 ITD/FLT3 wildtype ratios. Examples 
of sensitive and specific custom-made FLT3 ITD NGSbased mutation detection assays have been published. ${ }^{4,5}$

\section{Sequencing}

A selected number of fusion transcripts have been incorporated in the 2017 ELN recommendations and classify AML patients into various risk categories. ${ }^{1}$ Although the majority of these gene rearrangements are revealed by cytogenetic analyses, molecular approaches are often complementary. Fusion transcript detection was long limited to those transcripts for which standardized assays, generally real-time quantitative PCR, were available. However, all fusion transcripts relevant for risk stratification of AML can now be detected in a single assay with RNA-based NGS (RNA sequencing). Given that at least one of the partner genes involved in all clinically relevant fusions is known, RNA sequencing analysis can focus on these specific genes, leaving the possibility of revealing novel fusion partners. Such targeted RNA sequencingbased assays are commercially available. However, since the number of clinically relevant fusion transcripts is limited, one could also consider developing customized methods in which the AML-associated transcripts are amplified by (multiplex) PCR and subsequently sequenced by NGS. ${ }^{6}$ Again, proper validation of these assays at the local site is essential.

Currently, library preparation and sequencing with amplicon-based NGS assays usually require several days, whereas analyses of the limited number of 2017 ELN genes can be done rather quickly. The introduction of novel NGS machines with faster turnaround times, such as the Illumina iSeq100, and the development of customized assays now enable fast library preparation and overnight sequencing, thus allowing for a quick return of test results to the clinic. This is of particular interest when targetable mutations, such as those in FLT3, IDH1 or $I D H 2$, are needed for selection of the appropriate drug for front-line AML therapy or for relapsed patients with a high disease burden.

Since most of the clinically relevant mutations in myeloid malignancies are known, targeted sequencing is currently the method of choice. However, it can be foreseen, when turnaround times and costs are reduced, that whole exome or whole genome sequencing will become the standard approach to genomic characterization of AML at diagnosis. The use of whole exome or whole genome sequencing will allow identification of all somatic coding mutations, including those that are targetable but less frequently present in AML. Moreover, one can prioritize analysis of key AML genes first, such that initial results regarding the clinically most relevant genes can be obtained with a short turnaround and more comprehensive genomic profiling can follow later. Furthermore, whole genome sequencing allows identification of novel biomarkers located outside of protein coding regions, which may be useful not only for proper assessment of the prognosis but also for detection of minimal residual disease (MRD) in AML as they can be used to identify and follow leukemic clones regardless of their role in AML initiation and maintenance.

\section{Minimal residual disease}

Our improved understanding of the molecular landscape of AML has resulted in better treatment decisions at the time of complete remission after induction treatment.
Although the majority of AML patients achieve complete remission, many eventually relapse. Thus, there is still a great need for adequate prediction for subsequent relapse to adapt treatment accordingly and improve the outcomes of patients at high risk of relapse. MRD detection has already proven to have substantial value in predicting relapse and overall survival when applied to AML in complete remission but the use of molecular enumeration of $\mathrm{MRD}$ has been limited to only specific, molecularly defined subtypes of AML..$^{7-9}$ By contrast, flow cytometric analysis of MRD can be done in nearly all AML patients, but is operator- and center-dependent and there is no centrally agreed approach to enumerate flow-based MRD in AML. NGS enables MRD detection by measuring all mutations, including patient-specific persistent mutations, in complete remission. In fact, it has recently been shown that molecular MRD detection by NGS is applicable to virtually every newly diagnosed AML patient because of the frequent prevalence of multiple molecular aberrations among patients with AML. ${ }^{10-13}$ However, MRD detection based on NGS must overcome several challenges before it can be reliably introduced into clinical practice.

The known oligoclonality of the disease at diagnosis has a clear impact on MRD detection. Molecular markers in small AML subclones at diagnosis could easily be missed by panel-based NGS at lower depth. However, these small populations of cells may be selected during therapy and ultimately result in AML relapse. This issue could be overcome by sensitive detection of all possible mutations frequently present in myeloid malignancies. However, because of the relatively high error rates of current standard NGS technologies, reliable detection of a multitude of mutations at high sensitivities $(<0.01 \%)$ is not yet easily achieved. In fact, the currently high intrinsic error rates ( 1 to $0.1 \%$ ) impede sensitive MRD monitoring at later time points during therapy as well. At these time points certain targets present at diagnosis can be sequenced individually with single amplicons at high depth, but true residual mutations may still not be reliably discriminated from noise at levels below $0.1 \%$. Attempts should be made to improve the signal-to-noise ratios in order to detect low-level variants accurately. ${ }^{14}$ Genomic DNA isolated from bone marrow, peripheral blood or mononuclear cells is generally of high quality, but noise in NGS is subsequently introduced at different levels during library preparation and sequencing. ${ }^{14}$ The rate of sequencing artefacts can be reduced biochemically, e.g., by using proof-reading polymerases, or computationally; however, these corrections are only modest and cannot attenuate errors/artefacts entirely. Alternative strategies should be explored. Recently, various error-corrected NGS methodologies using molecular barcoding have been introduced. ${ }^{14}$ Error-corrected sequencing is based on barcoding the individual DNA molecules used for NGS library preparation. Using the unique sequence tag all derivative reads, which arise from a common founder, can be recognized after computational NGS, which enables removal of PCR duplicates and false mutation calls. These approaches and protocols ${ }^{15}$ have been shown to increase the specificity of low-frequency mutation detection. ${ }^{14}$ However, whether error-corrected sequencing will improve MRD detection by NGS in AML remains to be demonstrated in large cohorts of AML. 
Recently, several studies addressed NGS-based MRD detection in relatively large AML cohorts from clinical trials, all demonstrating that NGS-based MRD has a profound prognostic impact in patients with AML..$^{11-13,16}$ In these studies persistent mutations in complete remission were measured with gene panels, ${ }^{11}$ capture-based deep sequencing ${ }^{10,12,16}$ or targeted sequencing. ${ }^{13}$ The ampliconbased approach was specifically designed for MRD detection by including error-correction, ${ }^{13}$ which suggests that previous NGS-based MRD studies were not yet optimal. The results of these initial studies do not allow any firm conclusions to be drawn with regard to the superiority of error-corrected NGS for MRD detection in AML. However, the fact that NGS MRD has consistent prognostic value implies that technological improvements should be made in order to further optimize relapse prediction in AML, assuming that in these initial studies minor AML MRD clones were missed in complete remission. The ELN MRD Working Group is currently aiming to improve and harmonize methodologies for NGS-based detection of MRD in AML.

Another successful approach to correct for noise is to use a site-specific error model with a sufficiently large set of reference samples without mutations. ${ }^{11}$ In such a model the distribution of variants is determined in a reference set without mutations, for example, remission samples. MRD is subsequently defined by those mutations, such as the ones present at diagnosis, which are statistically significantly different from the distribution of variants in the reference set. In this case the detection sensitivity of mutations is variable and dependent on the average coverage for that specific locus for all samples, the observed error variance of the site-specific variant in the reference set (a high variance results in decreased detection sensitivity) and the number of control samples available. A major drawback of this approach is that a set of reference samples to determine MRD has to be available. MRD measurement in a single sample without the dependence of a large reference set is obviously a preferred method since it will be more easily implemented in clinical practice.

\section{Clonal hematopoiesis}

In the initial NGS-based MRD studies ${ }^{11-13,16}$ it became clear that gene mutations persisting in complete remission that are well-known to be associated with clonal hematopoiesis ${ }^{17,18}$ do not have an impact on the risk of relapse, despite the fact that they are among the most common disease-initiating drivers of AML. As a result of high-dose chemotherapy, AML patients with these mutations are apparently brought back into a state of clonal hematopoiesis, in which mutations occurring late in leukemogenesis are irradiated, but mutations also found in clonal hematopoiesis persist. It is clear that these persisting mutations, also known as clonal mutations of indeterminate potential, add another layer of complexity to $\mathrm{MRD}$ detection in AML.

In studies of molecular MRD, clonal hematopoiesisrelated mutations in DNMT3A, TET2 and ASXL1 (DTA) were considered clonal hematopoiesis rather than residual leukemia. Besides acquired mutations in DTA, other well-known pathogenic mutations such as those in TP53, $P P M 1 D, J A K 2, C B L, S R S F 2$ and SF3B1 are involved in clonal hematopoiesis, however, at lower incidence. ${ }^{17,18}$ In fact, many of these mutations also persist in complete remission with high variant allele frequencies. It needs to be determined whether and, if so, to what extent persisting mutations other than those in DTA represent true residual leukemia or clonal hematopoiesis, respectively with and without an increased risk of relapse. In a disease as heterogeneous as AML these analyses will require large cohorts of patients. Thus, while the recent developments in NGS-based MRD detection represent major steps forward in predicting relapse, they remain imperfect. It is expected that a better distinction between clonal hematopoiesis and residual leukemia will improve prediction of AML patients at higher risk of relapse.

How can the discrimination between true residual leukemia and clonal populations of cells be improved? The numbers of AML patients included in the initial studies precluded detailed analyses of rare mutations and indepth analysis of common non-DTA mutations. It is conceivable that non-DTA mutations are a mixture of mutations representative of either true leukemia or clonal hematopoiesis. Improved discrimination of these two conditions by means of types of persisting mutations may have significant value with regards to relapse prediction. Along the same lines it has recently been shown that it may be feasible to discriminate clonal hematopoiesis from pre-AML in healthy individuals. ${ }^{19}$ Pre-AML cases were distinct from controls and had more mutations per sample, higher variant allele frequencies, indicating clonal expansion, and showed enrichment in specific genes. ${ }^{19}$ Similar approaches could possibly better differentiate clonal hematopoiesis from true leukemia after induction treatment.

\section{Sensitivity, timing and tissues for next-generation sequencing}

How does NGS-based MRD detection perform as compared to the 'golden standard', multiparameter flow cytometric MRD detection? There are only limited studies with a rigorous comparison between NGS- and multiparameter flow cytometric MRD detection..$^{11,16}$ These studies demonstrate that there is a $70 \%$ concordance with regard to MRD detection using the two technologies, and that those patients who are MRD-positive according to both techniques have the highest risk of developing an AML relapse..$^{11,16}$ Interestingly, however, those AML cases with discordant results from NGS and flow cytometry have adverse outcome, such that MRD positivity has value whether determined by flow cytometry, molecular techniques, or both. ${ }^{11,16}$ We need to improve both the sensitivity of our NGS assays and our understanding of the biology of clonal hematopoiesis after high-dose chemotherapy to resolve the discordant cases and determine whether we require both technologies or only one to enumerate MRD.

At which time point(s) should NGS-based MRD detection be carried out? In the majority of AML studies NGSbased MRD detection was performed after high-dose induction treatment. Although this time point may be most suitable for choosing the proper consolidation treatment, it is not known whether MRD assessment at other time points may be better prognostic indicators. Few studies have shown that MRD before and after consolidation, such as in the setting of allogenic transplantation, affects clinical outcome. . $^{12,16,20}$

A bone marrow biopsy is an invasive procedure that gives stress and physical discomfort to a patient and creates a risk of infection. Patients with chronic myeloid 
leukemia are monitored by measuring $B C R-A B L 1$ levels in peripheral blood. Likewise, in mutant NPM1 AML, response to treatment can be effectively ascertained in peripheral blood. Studies should be carried out to determine whether peripheral blood is also an alternative for NGS-based MRD monitoring in AML for a broader spectrum of molecular alterations.

\section{Conclusions}

NGS at diagnosis is essential for accurate risk stratification of AML patients according to the 2017 ELN recommendations and has now been implemented in many molecular diagnostic laboratories. Currently, the major limitations of the NGS-based methodology of detecting $\mathrm{MRD}$ are related to the limited sensitivity and specificity of the assays and the inability to discriminate correctly between residual leukemia and clonal hematopoiesis.
Improvements need to be made in these areas before NGS-based MRD detection can be successfully implemented in routine practice.

\section{Acknowledgments}

R.L.L. is on the supervisory board of Qiagen and is a scientific advisor to Loxo, Imago, C4 Therapeutics and Isoplexis, which each include an equity interest. He receives research support from and consulted for Celgene and Roche, he has received research support from Prelude Therapeutics, and he has consulted for Incyte, Novartis, Morphosys and Janssen. He has received honoraria from Lilly and Amgen for invited lectures and from Gilead for grant reviews.

\section{Funding}

This work was supported in part by MSKCC Support Grant/Core Grant P3O CA008748 and the Netherlands Organization for Health Research and Development ZonMw (846002002).

\section{References}

1. Dohner H, Estey E, Grimwade D, et al. Diagnosis and management of AML in adults: 2017 ELN recommendations from an international expert panel. Blood. 2017;129(4):424-447.

2. Onecha E, Linares M, Rapado I, et al. Novel deep targeted sequencing method for minimal residual disease monitoring in acute myeloid leukemia. Haematologica. 2018;104 (2):288-296

3. Murphy KM, Levis M, Hafez MJ, et al. Detection of FLT3 internal tandem duplication and D835 mutations by a multiplex polymerase chain reaction and capillary electrophoresis assay. J Mol Diagn. 2003;5 (2):96-102.

4. Schranz K, Hubmann M, Harin E, et al. Clonal heterogeneity of FLT3-ITD detected by high-throughput amplicon sequencing correlates with adverse prognosis in acute myeloid leukemia. Oncotarget. 2018;9(53): 30128-30145.

5. Levis MJ, Perl AE, Altman JK, et al. A nextgeneration sequencing-based assay for minimal residual disease assessment in AML patients with FLT3-ITD mutations. Blood Adv. 2018;2(8):825-831.

6. Dillon LW, Hayati S, Roloff GW, et al. Targeted RNA-sequencing for the quantification of measurable residual disease in acute myeloid leukemia. Haematologica. 2018;104(2):297-304
7. Hourigan CS, Gale RP, Gormley NJ Ossenkoppele GJ, Walter RB. Measurable residual disease testing in acute myeloid leukaemia. Leukemia. 2017;31(7):14821490.

8. Ivey A, Hills RK, Simpson MA, et al Assessment of minimal residual disease in standard-risk AML. N Engl J Med. 2016;374 (5):422-433.

9. Kronke J, Schlenk RF, Jensen $\mathrm{KO}$, et al. Monitoring of minimal residual disease in NPM1-mutated acute myeloid leukemia: a study from the German-Austrian acute myeloid leukemia study group. J Clin Oncol. 2011;29(19):2709-2716.

10. Klco JM, Miller CA, Griffith $M$, et al. Association between mutation clearance after induction therapy and outcomes in acute myeloid leukemia. JAMA. 2015;314 (8):811-822.

11. Jongen-Lavrencic M, Grob T, Hanekamp D et al. Molecular minimal residual disease in Acute Myeloid Leukemia. N Engl J Med. 2018;378(13):1189-1199.

12. Morita K, Kantarjian HM, Wang F, et al. Clearance of somatic mutations at remission and the risk of relapse in acute myeloid leukemia.. J Clin Oncol. 2018;36(18):17881797.

13. Thol F, Gabdoulline R, Liebich A, et al. Measurable residual disease monitoring by NGS before allogeneic hematopoietic cell transplantation in AML. Blood. 2018;132(16):1703-1713.
14. Salk JJ, Schmitt MW, Loeb LA. Enhancing the accuracy of next-generation sequencing for detecting rare and subclonal mutations. Nat Rev Genet. 2018;19(5):269-285.

15. Wong WH, Tong RS, Young AL, Druley TE. Rare event detection using error-corrected DNA and RNA sequencing. J Vis Exp. 2018;(138).

16. Getta BM, Devlin SM, Levine RL, et al Multicolor Flow Cytometry and Multigene Multicolor flow cytometry and multigene next-generation sequencing are complementary and highly predictive for relapse in acute myeloid leukemia after allogeneic transplantation. Biol Blood Marrow Transplant. 2017;23(7):1064-1071.

17. Genovese G, Kahler AK, Handsaker RE, et al. Clonal hematopoiesis and blood-cancer risk inferred from blood DNA sequence. N Engl J Med. 2014;371(26):2477-2487.

18. Jaiswal S, Fontanillas P, Flannick J, et al. Agerelated clonal hematopoiesis associated with adverse outcomes. N Engl J Med. 2014;371 (26):2488-2498.

19. Abelson S, Collord G, Ng SWK, et al Prediction of acute myeloid leukaemia risk in healthy individuals. Nature. 2018;559 (7714):400-404.

20. Kim T, Moon JH, Ahn JS, et al. Next-generation sequencing-based posttransplant monitoring of acute myeloid leukemia identifies patients at high risk of relapse. Blood. 2018;132(15):1604-1613. 